\title{
Point of View
}

\section{Should we be screening for ovarian cancer?}

\author{
Kanishka Karunaratne ${ }^{1}$ \\ Sri Lanka Journal of Obstetrics and Gynaecology 2011; 33: 68-69
}

Ovarian cancer carries the worst prognosis of all gynaecological cancers, with an overall five-year survival rate of around 30-40 percent. For women diagnosed with early-stage disease the five-year survival rate is over 80 percent $^{1}$. Majority of the ovarian cancers present in advanced stages due to vague symptoms. Advanced stage disease carries a poor fiveyear survival rate of around 15 percent. Ovarian cancer accounts for 4 percent of all cancers in women. In Sri Lanka the incidence is around 12 per 100000 and had shown an upward trend during the last two decades.

\section{Value of early diagnosis}

Most improvements in survival have been in women presenting with stage I and stage II disease. This suggests that early detection of ovarian cancer could greatly improve the prognosis. However, due to lack of specific symptomatology, early diagnosis is often difficult. Dispelling the myth that ovarian cancer is a 'silent killer' studies have shown that the symptoms are present in more than 90 percent of women with early disease and may be present up to 15 months before diagnosis ${ }^{2,3}$. These symptoms include persistent pelvic and abdominal pain, abdominal distention and bloating. However, the natural history of ovarian cancer is not well understood and there is, at present, no evidence that ovarian cancer screening can reduce the mortality.

\section{Potential screening tests}

There is no single effective screening test for ovarian cancers. The main strategy for screening includes both biochemical markers and transvaginal or pelvic ultrasound.

Transvaginal ultrasound has a high sensitivity but a low specificity for malignant lesions ${ }^{4}$. This results in many women requiring further investigations and undergoing potential unnecessary surgery.

The serum cancer antigen 125 (CA 125) has a poor sensitivity. The antigen is expressed in only

\footnotetext{
${ }^{1}$ Consultant Gynae-oncologist, National Cancer Institute, Maharagama, Sri Lanka.

E-mail:kushank@sltnet.lk
}

80 percent of ovarian tumours and in only 50-60 percent of stage I disease ${ }^{5}$. Furthermore, it is nonspecific in that it may be increased in the presence of other cancers as well as benign conditions including fibroids, diverticulitis, endometriosis and menstruation.

\section{Randomized controlled trials of multimodal screening (MMS)}

There are two ongoing studies designed to test the efficacy of MMS, the United Kingdom Collaborative Trial of Ovarian Cancer (UKCTOCS) in $\mathrm{UK}^{6}$ and the Prostate, Lung, Colorectal and Ovarian (PLCO) cancer screening trial in USA ${ }^{7}$. The UKCTOCS has randomly assigned more than 200,000 women to no screening, annual CA 125 followed by TVS as a second-lined test if risk is indicated by the CA 125 results, and annual TVS alone in the ratio 2:1:1.

The PLCO has recruited 74,000 postmenopausal women and is comparing a controlled group with a screened group undergoing primary screening with both CA 125 and TVS for three years, and then CA125 alone for a further two years. The results of both trials are expected in 2015.

\section{Interim trial results}

In the UKCTOCS, a recent report of the prevalence screen showed that, of the 98,308 women screened in either group, 942 had surgery ${ }^{6}$. Of these, 834 (47 in the MMS group and 787 USS group) had benign growths or normal ovaries. Of these, 2.9 percent had major complication of surgery. Overall, 8.7 women in USS group received surgery for every one woman in the TVS group. In the MMS group, of 97 surgical evaluations, 42 ovarian cancers were detected. Of these, 34 were primary invasive epithelial ovarian tumours and tubal cancers. In the USS group, of the 845 women undergoing surgery, 45 ovarian cancers resulted. In the MMS group, the overall sensitivity, specificity and positive predictive values for all primary invasive epithelial ovarian and tubal cancers were 89.5, 99.8 and 43.3 percent compared with 75.0, 98.2 and 2.8 percent for USS. The authors conclude that the results show that the 'screening strategies are feasible'. 
In the PLCO study, of the 28816 women tested 570 surgical interventions resulted, a total of 29 neoplasms being detected, of which 19 were epithelial invasive cancers ${ }^{7}$.

\section{Conclusion}

The results to date demonstrate the dilemma with all screening, in that early detection needs to be balanced against potentially treating many women unnecessarily. While the results are encouraging, particularly for MMS, it is not until the effect of ovarian cancer screening on mortality is known in 2015 that a population screening programme can be considered. The UKCTOCS will also provide comprehensive data on cost, acceptance, physical and psychological morbidity.

Until such time, improving awareness of the symptoms of ovarian cancer will be crucial in ensuring that women with ovarian cancer are diagnosed at an early stage. Although symptoms are non-specific, GPs and women need to be aware that there is increasing evidence that women with ovarian cancer do experience symptoms more frequently, severely and persistently than those without the disease ${ }^{8,9}$. Responding to these symptoms without delay is vital if we are to counter a disease that at present has a very poor prognosis.

\section{References}

1. Cancer Research UK. UK ovarian cancer statistics. 2009 (http://info.cancerresearchuk.org/cancerstats/types/ ovary; accessed 19 February 2009).

2. Goff BA, Mandel L, Muntz HG, et al. Ovarian carcinoma diagnosis: results of a national ovarian cancer survey. Cancer 2000; 89: 2068-75.

3. Lim A. Investigating the potential for expediting diagnosis of ovarian cancer via prompt symptom recognition and targeted screening. PhD thesis, Queen Mary University, London, 2009.

4. DePriestPD, van Nagell JR. Transvaginal ultrasound screening for ovarian cancer. Clin Obstet Gynecol 1992; 25: 40-4.

5. Jacobs I, Stabile I, Bridges J, et al. Multimodal approach to screening for ovarian cancer. Lancet 1988; 1: 268-71.

6. Menon U, Gentry-Maharaj A, Hallet R, et al. Sensitivity and specificity of multimodal and ultrasound screening for ovarian cancer: results of the prevalence screen of the UK Collaborative Trial of Ovarian Cancer Screening (UKCTOCS). Lancet Oncol 2009; 10: 327-40.

7. Buys S, Partridge E, Greene $\mathrm{M}$, et al. Ovarian cancer screening in the Prostate, Lung, Colorectal and Ovarian (PLCO) cancer screening trial: findings from the initial screen of a randomized trial. Am J Obstet Gynecol 2005; 193: 1630-9.

8. Hamilton $\mathrm{W}$, Peters $\mathrm{T}$, Bankhead C, et al. Risk of ovarian cancer in women with symptoms in primary care: a population based case-control study. BMJ 2009; 339: b2998.

9. Austoker J. Diagnosis of ovarian cancer in primary care. BMJ 2009; 339: b3286. 